Symposium

\title{
Association of Cardiovascular Risk Factors and Endothelial Dysfunction in Japanese Hypertensive Patients: Implications for Early Atherosclerosis
}

\author{
Tomoo FURUMOTO, Naotaka SAITO, Jie DONG, Taisei MIKAMI*, Satoshi FUJII, \\ and Akira KITABATAKE
}

\begin{abstract}
Although hypertension, hyperlipidemia, diabetes and smoking are known risk factors of atherosclerosis in Caucasians, their relative contributions to early atherosclerosis among Japanese are unknown. Decrease in flow-mediated dilation (FMD) of the brachial artery is a useful marker of endothelial dysfunction and early atherosclerosis. To evaluate the relative contribution of hypertension to early atherogenesis, we determined FMD, as well as plasma levels of tissue-type plasminogen activator (t-PA; a sensitive index of endothelial damage) and tumor necrosis factor (TNF)- $a$ and interleukin (IL)-6 (established markers of inflammation) in normotensive and hypertensive patients under treatment. FMD was significantly reduced as the number of risk factors increased, suggesting that accumulations of risk factors were related to endothelial dysfunction. FMD was reduced in hypertensives $(9.9 \pm 5.8$ (SD) \%) compared to normotensives $(14.6 \pm 7.6, p<0.01)$ despite good blood pressure control (139 $\pm 20 / 80 \pm 14 \mathrm{mmHg}$ in hypertensives). Nitroglycerine-induced endothelium-independent vasodilation was not altered in hypertensives (16.0 $6.3 \%$ ) as compared to normotensives (16.7 \pm 5.8). Plasma t-PA, TNF- $-\alpha$, and IL-6 levels were increased in hypertensives despite good blood pressure control. Thus, hypertension alone is a high risk for early atherosclerosis. Persistent endothelial damage and moderate inflammation may increase the risk of early atherosclerosis synergistically under the presence of hypertension in Japanese. (Hypertens Res 2002; 25: 475-480)
\end{abstract}

Key Words: inflammation, fibrinolysis, endothelial function, risk factors, atherosclerosis

\section{Introduction}

Although hypertension, hyperlipidemia, diabetes and smoking are known risk factors of atherosclerosis in Caucasians, their relative contributions to early atherosclerosis among Japanese have not been thoroughly investigated (1). Endothelial cells maintain vascular tone and structure (2). In response to shear stress derived from flowing blood, endothelium can release nitric oxide (NO), which causes smooth mus- cle cell relaxation (3). Flow-mediated dilation (FMD) of the brachial arteries, a useful noninvasive method to determine peripheral endothelial functions, is reduced in patients with hypertension and cardiovascular risk factors $(2,4)$.

With respect to other risk factors, inflammation has been implicated in vasculopathy (5). Plasma levels of tumor necrosis factor (TNF)- $\alpha$ and interleukin (IL)-6 are known to be elevated in atherosclerotic cardiovascular diseases (6), and the plasma level of tissue-type plasminogen activator ( $t$ PA), which plays a crucial role in modulating thrombosis

From the Department of Cardiovascular Medicine, Hokkaido University Graduate School of Medicine, Sapporo, Japan, and ${ }^{*}$ College of Medical Technology, Hokkaido University, Sapporo, Japan.

This work was supported in part by a Grant-in-Aid for Scientific Research from the Japanese Ministry of Education, Science, Sports and Culture (Tokyo, Japan), and by the grants from Takeda Medical Research Foundation (Osaka, Japan) and Mitsui Life Social Welfare Foundation (Tokyo, Japan).

Address for Reprints: Satoshi Fujii, M.D., Ph.D., Department of Cardiovascular Medicine, Hokkaido University Graduate School of Medicine, North 15, West 7, Kita-ku, Sapporo 060-8638, Japan. E-mail: sfujii@med.hokudai.ac.jp

Received December 20, 2001; Accepted in revised form February 12, 2002. 
and thrombolysis (7), is a sensitive index of endothelial damage (8) and is associated with risk of accelerated atherosclerosis (9).

Endothelial dysfunction may be reversed by antihypertensive therapy in experimental models of hypertension and in humans $(10,11)$. To evaluate the relative contribution of hypertension to early atherogenesis, we determined FMD, as well as plasma levels of t-PA (a sensitive index of endothelial damage) and TNF- $\alpha$ and IL-6 (established markers of inflammation) in normotensive and hypertensive patients under treatment.

\section{Methods}

\section{Study Population}

Patients were recruited from the Hokkaido University Hospital $(n=119)$. Written informed consent was obtained from all patients in accordance with the institutional ethical guidelines. Patients with a history of essential hypertension (pretreatment systolic blood pressure $\geqq 140 \mathrm{mmHg}$ and/or pretreatment diastolic blood pressure $\geqq 90 \mathrm{mmHg}$ ) that resulted in antihypertensive treatment (calcium blocker, $n=47 ; \beta$ blocker, $n=17$; angiotensin converting enzyme inhibitor, $n=17)$ were included in the hypertension group $(n=67)$. Control subjects were matched to hypertensive subjects according to age and gender $(n=52)$. Blood pressure was expressed as the mean of 3 different sphyngomanometric measurements, each performed on 3 separate days. The possibility of secondary hypertension was excluded by clinical and laboratory tests. Exclusion criteria also included heart failure, valvular heart disease, congenital heart disease, acute coronary syndrome and liver disorders. Clinical data, including risk factor assessment for coronary heart disease, biochemical data, and results of the present investigations, were recorded. A known history of smoking (current smokers included individuals who stopped smoking $<5$ years before the enrollment) was recorded. Body mass index (BMI) was calculated as the ratio of weight $(\mathrm{kg})$ to the square of height $\left(\mathrm{m}^{2}\right)$. Routine lipid estimation was performed, including estimation of total cholesterol, triglyceride (TG) and high-density lipoprotein (HDL) levels. Subjects with an elevated serum total cholesterol ( $>220 \mathrm{mg} / \mathrm{dl}$ ) or subjects with a history of elevated serum total cholesterol that resulted in cholesterollowering treatment were considered to have a positive history of hyperlipidemia. No subject had documented familial hyperlipidemia. Subjects with an elevated fasting plasma glucose ( $>126 \mathrm{mg} / \mathrm{dl}$ ) or subjects with a history of elevated fasting plasma glucose that resulted in anti-diabetes treatment were considered to have a positive history of diabetes mellitus.

\section{Sonographic Measurements}

Sonographic measurements were performed in 62 patients
(25 normotensives and 37 hypertensives). A 5-10 $\mathrm{MHz}$ high-resolution linear phased array ultrasound transducer (ATL HDI 3000, Tokyo, Japan) was used to image the dominant arm brachial artery longitudinally above the antecubital fossa. After an overnight fast, patients rested for $10 \mathrm{~min}$ in a quiet room. All vasodilating drugs except sublingual nitroglycerin spray were discontinued at least $18 \mathrm{~h}$ before the study. After the baseline image was obtained, a blood pressure cuff was inflated to $200 \mathrm{mmHg}$ on the forearm for $5 \mathrm{~min}$ and then released. Images were obtained every $30 \mathrm{~s}$ after cuff dilation and FMD was used as a measure of endothelium-dependent vasodilation. The brachial artery was then allowed to return to normal (10 $\mathrm{min})$, and repeated baseline images were obtained. After administration of $0.3 \mathrm{mg}$ sublingual nitroglycerin spray, the brachial artery was imaged for the ensuing $5 \mathrm{~min}$ for measurement of maximal vasodilation. Images were coded and recorded on VHS videotape for subsequent blinded analysis. The arterial diameter was measured in $\mathrm{mm}$ as the distance between the anterior and the posterior wall media-adventitial interface at end-diastole coincident with the $\mathrm{R}$ wave on the electrocardiogram at 2 sites along the artery and for 3 cardiac cycles, and expressed as the average of these 6 measurements. The average arterial diameter was used to calculate the percent diameter change of the brachial artery in response to reactive hyperemia or nitroglycerin. The intraobserver and interobserver variabilities for repeated measurements were $3.5 \%$.

\section{Blood Sampling and Laboratory Assays}

Morning blood samples were taken after an overnight fast. The samples were drawn into plastic tubes containing 1: 9 volumes of $0.103 \mathrm{~mol} / \mathrm{l}$ trisodium citrate and centrifuged at $3,000 \times \mathrm{g}$ for $15 \mathrm{~min}$. Aliquots of plasma were stored at $-80^{\circ} \mathrm{C}$ until analysis. Personnel blinded to the clinical data performed the laboratory measurements. Fasting plasma glucose was measured by the glucose dehydrogenase method, and immunoreactive insulin (IRI) was measured by enzymelinked immunosorbent assay (ELISA). Homeostasis model assessment (HOMA), defined as IRI $(\mu \mathrm{U} / \mathrm{ml}) \times$ fasting plasma glucose $(\mathrm{mg} / \mathrm{dl}) / 405$, was used as an index of insulin sensitivity. The plasma level of t-PA antigen was measured by ELISA (Biopool, Umeå, Sweden). Plasma TNF- $\alpha$ was measured by high sensitivity immunoassay (R\&D Systems, Minneapolis, USA). The sensitivity of the assay is 0.11 $\mathrm{pg} / \mathrm{ml}$. The plasma concentration of IL- 6 was measured by enzyme-linked immunoassay (R\&D Systems), which can detect values as low as $0.094 \mathrm{pg} / \mathrm{ml}$. The cytokine levels in the present study were within the detection limit of the assay. All assays were performed in duplicate and the intra- and interassay variabilities were less than $10 \%$.

\section{Statistical Evaluation}

Data were expressed as the mean \pm SD. A $\chi^{2}$ analysis was 
Table 1. Characteristics of Patients

\begin{tabular}{lcc}
\hline & $\begin{array}{c}\text { Hypertensives } \\
(n=67)\end{array}$ & $\begin{array}{c}\text { Normotensives } \\
(n=52)\end{array}$ \\
\hline Age (years) & $63.7 \pm 10.9$ & $59.4 \pm 14.8$ \\
Male (\%) & 62.7 & 73.1 \\
Blood pressure (mmHg) & & \\
$\quad$ Systolic & $139 \pm 20$ & $118 \pm 13^{* *}$ \\
$\quad$ Diastolic & $80 \pm 14$ & $71 \pm 11^{* *}$ \\
Total cholesterol (mg/dl) & $186 \pm 32$ & $196 \pm 39$ \\
TG (mg/dl) & $153 \pm 75$ & $123 \pm 60^{*}$ \\
HDL (mg/dl) & $51 \pm 14$ & $56 \pm 17$ \\
BMI (kg/m $\left.{ }^{2}\right)$ & $25.1 \pm 4.0$ & $23.3 \pm 2.9^{* *}$ \\
Diabetes $(\%)$ & 55.2 & $20.3^{* *}$ \\
HOMA index & $1.66 \pm 1.48$ & $1.39 \pm 2.02$ \\
Smoking (\%) & 40.3 & 50 \\
Brachial artery diameter (mm) & $4.26 \pm 0.67$ & $4.20 \pm 0.67$ \\
\hline TG, triglyceride; HDL, high-density lipoprotein; BMI, body & ${ }^{*} p<0.05$, \\
mass index; HOMA, homeostasis model assessment. ${ }^{*} p$ &
\end{tabular}

used to determine differences in categorical variables. An unpaired Student's $t$-test was used to assess differences in continuous variables. Values of $p<0.05$ were considered to indicate statistical significance.

\section{Results}

\section{Brachial Artery FMD and Nitroglycerin-Induced Dila- tion}

The characteristics of the normotensive and hypertensive patients are summarized in Table 1. The blood pressure in the hypertensive group was well controlled. No difference in brachial artery diameter was noted between the two groups. FMD was significantly lower in hypertensives $(9.9 \pm 5.8 \%)$ than in normotensives $(14.6 \pm 7.6 \%, p<0.05)$ (Fig. 1A). Endothelium independent vasodilation was not significantly lower in hypertensives $(16.0 \pm 6.3 \%)$ than in normotensives $(16.7 \pm 5.8 \%)$.

\section{Markers of Fibrinolysis and Inflammation}

The plasma t-PA antigen level was higher in hypertensives than in normotensives $(10.4 \pm 4.2$ vs. $8.4 \pm 4.2 \mathrm{ng} / \mathrm{ml}, p<$ $0.05)$, indicating continued endothelial damage despite blood pressure reduction (Fig. 2A). The plasma TNF- $\alpha$ level was higher in hypertensives than in normotensives (2.6 \pm 1.5 vs. $2.1 \pm 1.4 \mathrm{pg} / \mathrm{ml}, p<0.05)$, indicating persistent inflammation (Fig. 2A). However, the plasma IL-6 level was not significantly different between hypertensives and normotensives (1.8土 1.2 vs. $1.5 \pm 1.2 \mathrm{pg} / \mathrm{ml}$ ).
A

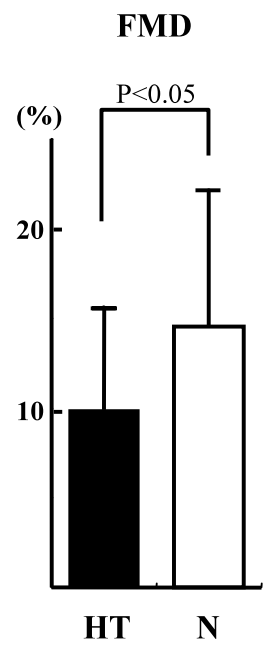

B

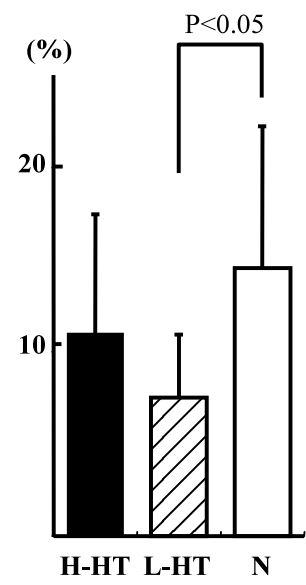

Fig. 1. (A) Comparison of flow mediated dilation (FMD) in normotensives $(N: \mathrm{n}=52)$ and hypertensives $(H T: \mathrm{n}=67)$. (B) Comparison of FMD in normotensives $(N: \mathrm{n}=52)$, low hypertensives (L-HT: systolic blood pressure 130 $\mathrm{mmHg}$ and diastolic blood pressure $\leqq 85 \mathrm{mmHg}, \mathrm{n}=22)$ and high hypertensives (H-HT: systolic blood pressure $>130$ $\mathrm{mmHg}$ and/or diastolic blood pressure $>85 \mathrm{mmHg}, \mathrm{n}=45$ ). Values are expressed as the mean $\pm S D$.

\section{Control of Blood Pressure and FMD}

The hypertensives were divided into the following two subgroups based on their level of blood pressure control. Blood pressure was insufficiently controlled in high hypertensives (H-HT; systolic blood pressure $>130 \mathrm{mmHg}$ and/or diastolic blood pressure $>85 \mathrm{mmHg}$ ), and well controlled in low hypertensives (L-HT; systolic blood pressure $\leqq 130 \mathrm{mmHg}$ and diastolic blood pressure $\leqq 85 \mathrm{mmHg}$ ). The clinical characteristics in terms of age, gender, cholesterol, TG, HDL, BMI, HOMA, brachial artery diameter and prevalence of diabetes and smoking did not differ between the H-HT and LHT groups (results not shown). Calcium blocker was used in $67 \%$ of H-HT and $71 \%$ of L-HT subjects. Angiotensin converting enzyme inhibitor was used in $28 \%$ of $\mathrm{H}-\mathrm{HT}$ and $19 \%$ of L-HT and $\beta$ blocker in $22 \%$ of H-HT and $33 \%$ of L-HT subjects. The average blood pressure in the L-HT group $(119 \pm 8 / 70 \pm 8 \mathrm{mmHg})$ was not significantly higher than that in normotensives $(118 \pm 13 / 71 \pm 11 \mathrm{mmHg})$. However, the blood pressure of the H-HT group was significantly higher than that of either normotensives or L-HT subjects (149 \pm $17 / 85 \pm 14, p<0.01)$. Despite the good blood pressure control in the L-HT group, the FMD of this group was significantly lower than that in normotensives (Fig. 1B). And the plasma level of TNF- $\alpha$ in the L-HT group was significantly higher than that in normotensives (Fig. 2B). 


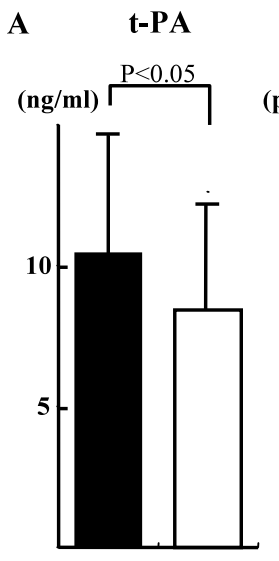

HT N
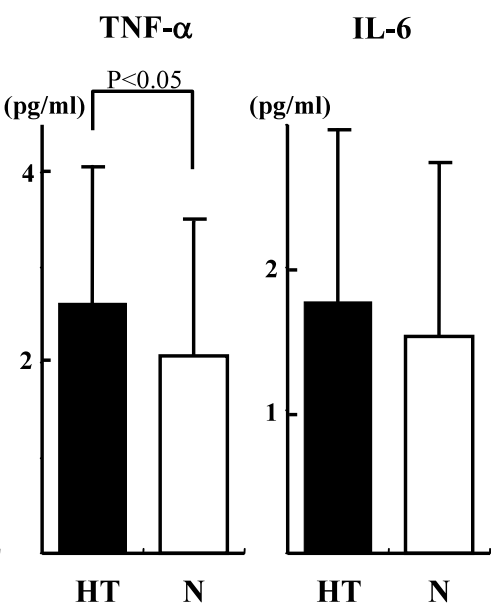

B

t-PA

TNF- $\alpha$

IL-6
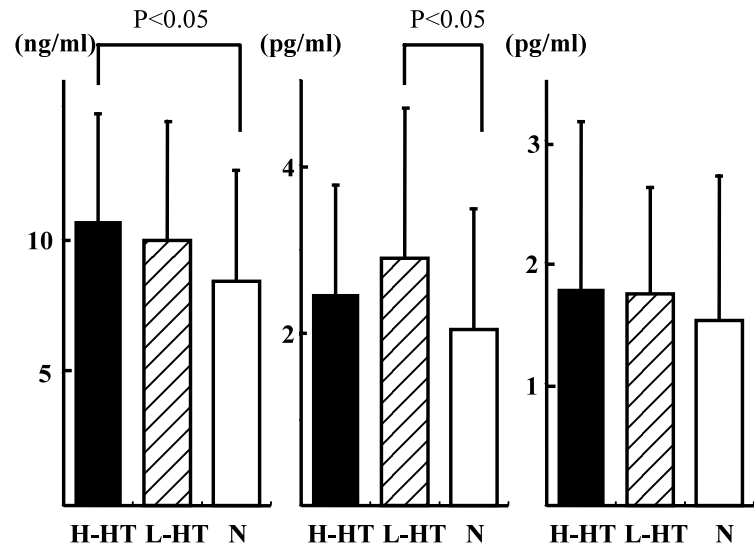

Fig. 2. (A) Comparison of plasma levels of $t-P A, T N F-\alpha$ and $I L-6$ levels in normotensives $(N: \mathrm{n}=52)$ and hypertensives $(H T$ : $\mathrm{n}=67)$. (B) Comparison of plasma levels of $t-P A, T N F-\alpha$ and IL-6 in normotensives $(N: \mathrm{n}=52)$, low hypertensives $(L-H T$ : systolic blood pressure $\leqq 130 \mathrm{mmHg}$ and diastolic blood pressure $\leqq 85 \mathrm{mmHg}, \mathrm{n}=22)$ and high hypertensives $(H$-HT: systolic blood pressure $>130 \mathrm{mmHg}$ and/or diastolic blood pressure $>85 \mathrm{mmHg}, \mathrm{n}=45$ ). Values are expressed as the mean $\pm S D$.

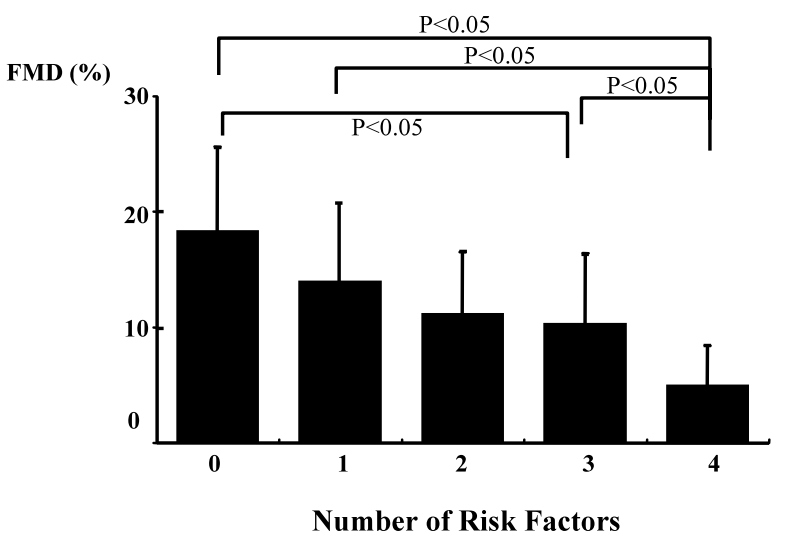

Fig. 3. Relationship between flow mediated dilation (FMD) and total number of risk factors. Risk factors include hypertension, hyperlipidemia, diabetes mellitus and smoking. Values are expressed as the mean $\pm S D$.

\section{Number of Risk Factors and FMD}

FMD was significantly reduced as the total number of risk factors increased (Fig. 3). Furthermore, hypertensives without hyperlipidemia, diabetes or a smoking habit exhibited significantly lower FMD compared to normotensives (Fig. 4). Among hypertensives, addition of one or two other risk factors did not further reduce FMD (Fig. 4). There was no difference in endothelium-independent relaxation between normotensives and hypertensives regardless of the number of risk factors. Among low-risk hypertension patients, in whom the only risk factors were hypertension or hypertension plus one other risk factor, plasma levels of t-PA, TNF- $\alpha$ and IL- 6 were moderately elevated in hypertensives as compared to

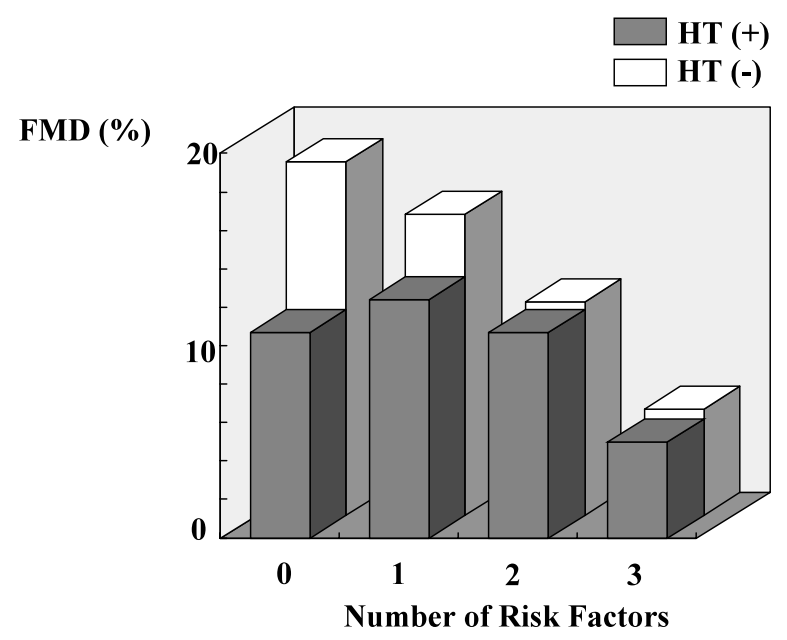

Fig. 4. Relationship between flow mediated dilation (FMD) and total number of risk factors (hyperlipidemia, diabetes and smoking) in subjects with or without hypertension. Values are expressed as the mean $\pm S D$.

normotensives (Fig. 5).

\section{Discussion}

FMD is known to be reduced in hypertensives $(12,13)$. In this study, FMD was lower in hypertensives despite good control of blood pressure, suggesting that a reduction of shear stress as a result of a decline in blood pressure may not be sufficient to fully restore endothelial function. There was no difference in endothelium-independent vasodilation between hypertensives and normotensives, suggesting that there may be no disruption of endothelium-independent 


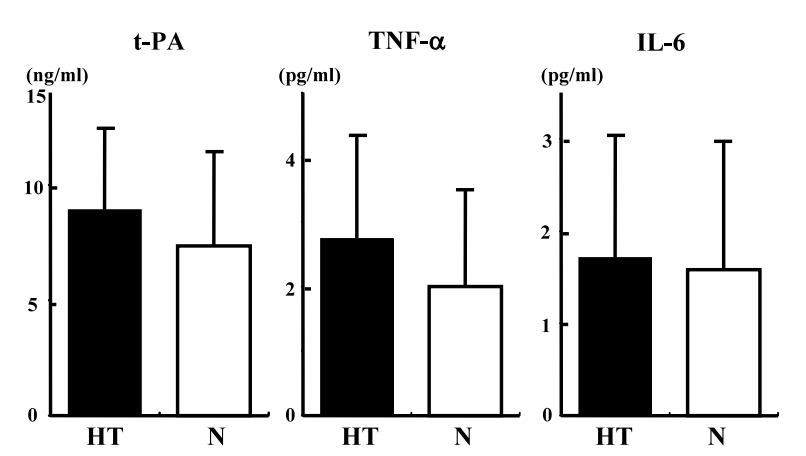

Fig. 5. Comparison of plasma levels of t-PA, TNF- $\alpha$ and IL-6 in normotensives $(N)$ and low-risk hypertensives (HT), in whom the only risk factors were hypertension or hypertension plus one other risk factor (hyperlipidemia, diabetes or smoking). Values are expressed as the mean士 SD.

vasodilation in hypertension.

In addition to decreased FMD, hypertensives exhibited higher plasma TNF- $\alpha$ levels than normotensives. Secretion of proinflammatory cytokines is upregulated in monocytes from hypertensives (14). Although TNF- $\alpha$ and IL-6 are the major determinants of acute phase protein production (15), their roles have not been thoroughly investigated in hypertensives. Inflammatory cytokines impair endothelium-dependent dilatation in human veins in vivo (16), and vascular inflammatory responses are implicated in hypertensioninduced end-organ damage (17). Thus, mild inflammatory reaction in hypertensives might be a useful index of impairment in vascular reactivity.

The level of t-PA antigen was increased in hypertensives in the present study, reflecting endothelial damage. TNF- $\alpha$ modulates the fibrinolytic system in the endothelium (18) and IL-6 induced by TNF- $\alpha$ has procoagulant properties (19). The results of a previous study indicate that there may be a close link between fibrinolysis and atherogenic metabolic derangement (20). An increased t-PA level may reflect chronic endothelial stimulation by thrombin and endothelial damage during vasculopathy. The results of a recent study by Newby et al. indicate that upregulation of basal t-PA release and subsequent depletion of endothelial t-PA stores play a role in coronary atherosclerosis (21).

Even a mild inflammatory reaction coupled with an alteration of fibrinolysis may disturb endothelial regulation of vascular tone in the arterial circulation in essential hypertension. In the present study, although both groups had similar blood pressure levels, the L-HT group had lower FMD than the normotensive subjects, suggesting that FMD might not have been fully improved by medication. TNF- $\alpha$ is linked to hypertension and angiotensin II is implicated in TNF- $\alpha$ regulation (22). Further, the use of angiotensin enzyme inhibitors is reported to improve FMD (23). The frequent use of angiotensin converting enzyme inhibitors in the H-HT group may partly explain the tendency for H-HT subjects to show slightly higher FMD and slightly lower plasma TNF- $\alpha$ levels compared to L-HT subjects. Alterations in endothelial function may provide a link between systemic inflammation and hypertension.

In this study, the presence of hypertension alone-i.e., without hyperlipidemia, diabetes or smoking — was associated with reduced FMD, suggesting that hypertension per se is a very important risk factor among Japanese. Furthermore, addition of other risk factors only moderately reduced FMD in hypertensives, suggesting that future treatment of hypertension may need to include anti-inflammatory therapeutic strategies directed at reducing inflammation (24). Because the cross-sectional nature of the present study did not permit analysis of causal inferences, further studies on fibrinolytic factors and inflammatory reactions will be needed to clarify the pathogenesis of early atherosclerosis in hypertension and to determine alternative preventive measures against clinical cardiovascular events. Accurate noninvasive evaluation of peripheral vascular function $(25,26)$ and morphology (27) may become useful for predicting future cardiovascular events in Japanese hypertensives.

In conclusion, impairment of FMD reflects a risk factor burden, and hypertension alone indicates a high-risk status for early atherosclerosis. Persistent endothelial damage and moderate inflammation may increase the risk of early atherosclerosis synergistically under the presence of hypertension in Japanese.

\section{Acknowledgements}

The authors thank Dr. Fumihiro Sata (Department of Preventive Medicine, Hokkaido University Graduate School of Medicine) for his statistical assistance and Ms. Akiko Aita and Ms. Mizuho Kasai for their technical support.

\section{References}

1. Muratani H, Kimura Y, Fukiyama K, et al: Control of blood pressure and lifestyle-related risk factors in elderly Japanese hypertensive subjects. Hypertens Res 2000; 23: 441-449.

2. Celermajer DS: Endothelial dysfunction: Does it matter?: Is it reversible? J Am Coll Cardiol 1997; 30: 325-333.

3. Joannides R, Haefeli WE, Linder L, et al: Nitric oxide is responsible for flow-dependent dilatation of human peripheral conduit arteries in vivo. Circulation 1995; 91: 1314-1319.

4. Celermajer D, Sorensen K, Gooch V, et al: Non-invasive detection of endothelial dysfunction in children and adults at risk of atherosclerosis. Lancet 1992; 340: 1111-1115.

5. Ross R: Atherosclerosis-an inflammatory disease. $N$ Engl J Med 1999; 340: 115-126.

6. Mehta JL, Saldeen TG, Rand K: Interactive role of infection, inflammation and traditional risk factors in atherosclerosis and coronary artery disease. J Am Coll Cardiol 1998; 31: $1217-1225$.

7. Sobel BE: Insulin resistance and thrombosis: a cardiolo- 
gist's view. Am J Cardiol 1999; 84: 37J-41J.

8. Ridker PM, Vaughan DE, Stampfer MJ, Manson JE, Hennekens $\mathrm{CH}$ : Endogenous tissue-type plasminogen activator and risk of myocardial infarction. Lancet 1993; 341: 1165-1168.

9. Sobel BE, Woodcock-Mitchell J, Schneider DJ, Holt RE, Marutsuka K, Gold H: Increased plasminogen activator inhibitor type 1 in coronary artery atherectomy specimens from type 2 diabetic compared with nondiabetic patients: a potential factor predisposing to thrombosis and its persistence. Circulation 1998; 97: 2213-2221.

10. Luscher TF, Vanhoutte PM, Raij L: Antihypertensive treatment normalizes decreased endothelium-dependent relaxation in rats with salt-induced hypertension. Hypertension 1987; 9: 193-197.

11. Creager MA, Roddy MA: Effects of captopril and enalapril on endothelial function in hypertensive patients. Hypertension 1994; 24: 499-505.

12. Panza JA, Quyyumi AA, Brush JE Jr, Epstein SE: Abnormal endothelium-dependent vascular relaxation in patients with essential hypertension. $N$ Engl J Med 1990; 323: 22-27.

13. Taddei S, Virdis A, Ghiadoni L, Magagna A, Salvetti A: Vitamin C improves endothelium-dependent vasodilation by restoring nitric oxide activity in essential hypertension. Circulation 1998; 97: 2222-2229.

14. Dörffel Y, Lätsch C, Stuhlmüller B, et al: Preactivated peripheral blood monocytes in patients with essential hypertension. Hypertension 1999; 34: 113-117.

15. Anderson JL, Muhlestein JB, Carlquist J, et al: Randomized secondary prevention trial of azithromycin in patients with coronary artery disease and serological evidence for Chlamydia pneumoniae infection: the Azithromycin in Coronary Artery Disease: Elimination of Myocardial Infection with Chlamydia (ACADEMIC) study. Circulation 1999; 99: 1540-1547.

16. Bhagat K, Vallance P: Inflammatory cytokines impair endothelium-dependent dilatation in human veins in vivo. Circulation 1997; 96: 3042-3047.

17. Muller DN, Mervaala EMA, Schmidt F, et al: Effect of bosentan on NF- $\kappa \mathrm{B}$, inflammation, and tissue factor in angiotensin II-induced end-organ damage. Hypertension 2000; 36: 282-290.
18. van Hinsbergh VW, Vermeer M, Koolwijk P, Grimbergen J, Kooistra T: Genistein reduces tumor necrosis factor alpha-induced plasminogen activator inhibitor-1 transcription but not urokinase expression in human endothelial cells. Blood 1994; 84: 2984-2991.

19. Sancéau J, Kaisho T, Hirano T, Wietzerbin J: Triggering of the human interleukin- 6 gene by interferon- $\gamma$ and tumor necrosis factor- $\alpha$ in monocytic cells involves cooperation between interferon regulatory factor- $1, \mathrm{NF} \kappa \mathrm{B}$, and SP1 transcription factors. J Biol Chem 1995; 270: 2792027931.

20. Sobel BE: Increased plasminogen activator inhibitor-1 and vasculopathy: a reconcilable paradox. Circulation 1999; 99: 2496-2498.

21. Newby DE, McLeod AL, Uren NG, et al: Impaired coronary tissue plasminogen activator release is associated with coronary atherosclerosis and cigarette smoking: direct link between endothelial dysfunction and atherothrombosis. Circulation 2001; 103: 1936-1941.

22. Togashi N, Ura N, Higashiura K, Murakami H, Shimamoto $\mathrm{K}$ : The contribution of skeletal muscle tumor necrosis factor-alpha to insulin resistance and hypertension in fructosefed rats. J Hypertens 2000; 18: 1605-1610.

23. Anderson TJ, Elstein E, Haber H, Charbonneau F: Comparative study of ACE-inhibition, angiotensin II antagonism, and calcium channel blockade on flow-mediated vasodilation in patients with coronary disease (BANFF study). $J \mathrm{Am}$ Coll Cardiol 2000; 35: 60-66.

24. Peeters AC, Netea MG, Kullberg BJ, Thien $\mathrm{T}$, van der Meer JW: The effect of renin-angiotensin system inhibitors on pro- and anti-inflammatory cytokine production. Immunology 1998; 94: 376-379.

25. Morise T, Horita M, Honda N, et al: Noninvasive, continuous evaluation of peripheral vascular resistance in humans. Hypertens Res 2000; 23: 15-19.

26. Hashimoto M, Kozaki K, Eto M, et al: Association of coronary risk factors and endothelium-dependent flow-mediated dilatation of the brachial artery. Hypertens Res 2000; 23: 233-238.

27. Kato J, Aihara A, Kikuya M, et al: Risk factors and predictors of coronary arterial lesions in Japanese hypertensive patients. Hypertens Res 2001; 24: 3-11. 